\title{
Prognosis of patients with late spontaneous recanalization of the atherosclerotic occlusion of internal carotid arteries: A pilot case series
}

\author{
HONGLIANG WU, YING LIU, BING LI, HUILONG ZHANG and CHUANYU LIU \\ Department of Neurology, The Affiliated Yantai Yuhuangding Hospital of Qingdao University, \\ Yantai, Shandong 264000, P.R. China
}

Received March 29, 2018; Accepted July 26, 2018

DOI: $10.3892 /$ etm.2018.6714

\begin{abstract}
Late spontaneous recanalization (SR) of an occluded internal carotid artery (ICA) has been rarely reported and the prognosis of affected patients remains elusive. In the present study, the incidence of late SR of atherosclerotic occlusion of the ICA and associated outcomes were assessed in a single center. A total of 36 patients with ICA occlusion, who underwent carotid artery stenting or vertebral artery stenting in arteries other than the occluded ICA, were prospectively included and followed up for $\geq 18$ months. SR was diagnosed by color Doppler ultrasound imaging. The incidence of late $\mathrm{SR}$, the functional outcome and the incidence of adverse cardiovascular events in these patients were evaluated. During the follow-up, three patients had late SR of the occluded ICA $(8.3 \%)$. All patients had vascular events prior to the confirmation of late SR of the ICA. Subsequent to SR, the patients were clinically stable with preserved functional ability, based on the Modified Rankin Scale. Of these patients, one suffered from re-occlusion of the recanalized ICA without presenting with any novel significant symptoms. In conclusion, patients with late SR of the ICA appear to have preserved functional ability and favorable clinical outcomes. Large-scale cohorts are required to determine the clinical characteristics that contribute to the incidence of late SR of the ICA.
\end{abstract}

\section{Introduction}

Occlusion of the internal carotid artery (ICA) is common in patients with stroke or transient ischemic attack, and certain patients with chronic occlusion of the ICA may be asymptomatic. Furthermore, spontaneous recanalization (SR)

Correspondence to: Dr Ying Liu, Department of Neurology, The Affiliated Yantai Yuhuangding Hospital of Qingdao University, 20 Yuhuangding East Road, Yantai, Shandong 264000, P.R. China E-mail: lucy1143@126.com

Key words: internal carotid artery, cerebral artery occlusion, late spontaneous recanalization, cohort study may occur in patients with ICA occlusion, which may be further categorized as early or late SR, based on the cut-off duration of the mean hospitalization of these patients (usually two weeks). The results of previously published studies have indicated that early SR of an occluded ICA may occur within 1-2 $\mathrm{h}$ to two weeks after occlusion (1-4), and early SR of the ICA appears to be common in patients with extracranial ICA occlusion caused by arterial dissection (57-68\% of the evaluated cohorts) (3,5-8). However, the incidence of late SR of an occluded ICA has been reported to be markedly lower, with 2.0 and $3.8 \%$, respectively $(9,10)$. Although previously published studies and case reports have revealed that patients with late SR of the ICA tend to have a retained functional ability and favorable clinical outcomes and that it was possible to perform carotid endarterectomy in these patients, the etiologies of ICA occlusion have not been defined in detail, and the diverse causes ICA occlusion were not considered (10-15). Therefore, the present study aimed to evaluate the incidence of late SR or atherosclerotic occlusion of the ICA in a cohort of patients who underwent carotid artery stenting or vertebral artery stenting in arteries other than the occluded ICA. In addition, the functional ability, prognosis and clinical characteristics of patients with late SR of the ICA were explored.

\section{Materials and methods}

Study design and patients. This single-center, prospective cohort study was performed at the Department of Neurology of Yuhuangding Hospital (Yantai, China). A total of 36 patients who were admitted to the hospital to receive a procedure of carotid artery stenting or vertebral artery stenting between January 2012 and December 2016 were included in the screening for a possible existing atherosclerotic occlusion of the ICA. Patients were included based on the following criteria: i) Acute or chronic occlusion of the ICA identified by digital subtraction angiography (DSA); ii) one or more stenotic arteries were identified by DSA in addition to the occluded ICA, indicating that the stenosis and occlusion of the arteries were caused by atherosclerosis; iii) percutaneous arterial stenting was scheduled to be performed in arteries other than the occluded ICA. Patients with severe heart and lung disease, severe liver and kidney disease, blood system 
disease, a late stage of cancer or an expected survival time of $<3$ months were excluded. These patients received dual anti-platelet therapy with aspirin (100 mg/day) and clopidogrel $(75 \mathrm{mg} /$ day) for six months after interventional therapy. Statins were also prescribed. The late SR of the occluded ICA was defined as SR that occurred after two weeks of stroke. Since percutaneous cerebral arterial stenting was performed one month after the stroke, only late SR was detected. Carotid color Doppler ultrasound imaging and other routine examinations were performed during the follow-up. The study was approved by the Ethics Committee of the Affiliated Yantai Yuhuangding Hospital of Qingdao University (Yantai, China). All patients provided written informed consent to participate in the study.

Image assessment. The initial diagnosis of ICA occlusion was made by DSA during percutaneous cerebral arterial stenting. The DSA equipment used was obtained from Siemens Healthcare (model no. 1083004/Artis zee ceiling; Munich, Germany) and GE Healthcare (REF 2396420-2/Innova 3100-IQ; Little Chalfont, UK). Patients with SR were confirmed by carotid color Doppler ultrasound imaging, which was performed by an experienced physician during the vascular ultrasound examination. The Philips I-22 (Philips Medical Systems, Eindhoven, The Netherlands) with a linear multi-frequency range of 5.0-10.0 $\mathrm{MHz}$ was used.

Clinical assessments. Demographic and medical data, including age and sex, as well as history of smoking, drinking, hypertension, diabetes and atrial fibrillation, were collected from all patients. The severity of the stroke was assessed according to the National Institute of Health Stroke Scale within $24 \mathrm{~h}$ of admission (16). Blood lipids and fasting blood glucose were measured as part of the fasting biochemical profile determined on the morning after admission. Hemoglobin, blood platelets, prothrombin time, activated partial thromboplastin time and fibrinogen were simultaneously measured. The participants of the present study underwent a series of clinical and instrumental follow-ups for the detection of SR, functional ability and the incidence of adverse cardiovascular events (recurrent stroke, non-fatal myocardial infarction or death of vascular causes). Carotid color Doppler ultrasound imaging and other follow-up investigations were performed at 1 month \pm 7 days, 3 months \pm 15 days, 6 months \pm 15 days, 12 months \pm 15 days, 18 months \pm 15 days after stent placement. The Modified Rankin Scale (mRS) was used to evaluate the functional ability of these patients (17).

Statistical analysis. Continuous variables were expressed as the mean \pm standard deviation, if normally distributed. Categorical variables were expressed as frequencies. Comparisons for categorical baseline measurements were performed by Fisher exact test and for continuous, not normally distributed baseline data, by the Mann-Whitney U test. Univariate logistic regression was used to determine the association between conventional risk factors and the incidence of late SR of the ICA occlusion. STATA (version 14.0; StataCorp LLC, College Station, TX, USA) was used for the statistical analysis.

\section{Results}

Baseline characteristics of the patients. The baseline characteristics of the patients included in the present study are presented in Table I. Among these 36 patients, 33 (91.7\%) were male and the median age of the patients was 65 years (IQR, 60-72 years). Furthermore, of these patients, 18 (50\%) experienced an ischemic stroke at admission, while 18 (50\%) had a transient ischemic attack at admission. No statistically significant differences in the baseline characteristics were noted between the two groups of patients with or without SR potentially due to the small sample size of patients with late SR $(n=3)$. Furthermore, none of the baseline characteristics was associated with the occurrence of late SR according to univariate analysis (Table II).

Characteristics of the cerebral vascular artery. The characteristics of the cerebral vascular artery are presented in Table III. A total of 22 patients $(61.1 \%)$ with carotid artery occlusion were symptomatic and 24 patients $(68.6 \%)$ had an occlusion of the right ICA. Furthermore, 22 patients $(61.1 \%)$ had one or more vertebral stenotic arteries, 25 patients (69.4) had a carotid artery stenosis in addition to the occluded ICA and 15 patients (41.7) had one or more intracranial stenotic arteries.

Follow-up information of the patient cohort. Adverse cardiovascular events and their incidence are presented in Table III. Among the 36 patients, 16 (44.4) had vascular events. Among these 16 patients, $9(25 \%)$ had a recurrent stroke, $7(19.4 \%)$ had cardiovascular events and 2 died due to vascular events (5.6\%). At the end of the follow-up period, 5 patients with no spontaneous late recanalization had a $\mathrm{mRS}$ score of $\geq 3$. Among these patients with $\mathrm{mRS}$ score $\geq 3$, two patients died. Patients with SR appeared to have better functional ability, based on the Modified Rankin Scale. Due to the small sample size, statistical comparisons cannot be made.

Characteristics, follow-up information and outcomes of patients with late SR. Among these 36 patients, 3 (8.3\%, including two males) had late SR, which occurred at the 3rd, 16th and 18th month after the occluded ICA was confirmed. The characteristics and outcomes of these 3 patients are listed in Table IV. Furthermore, two male patients presented with moderately sized infarcts caused by occlusion of the right carotid artery, while a female patient presented with posterior circulation ischemia without infarction. These two male patients suffered from lacunar infarction immediately prior to the confirmation of SR, while the female patient suffered a non-fatal myocardial infarction prior to identification of SR. The SR of the ICA in the first patient was demonstrated by DSA and featured a string flow, and that in the second patient was demonstrated by MRA and was characterized by a winding thin line. MRA and DSA were recommended for the third patient, but the patient refused to undergo these procedures. After 6 months, the left proximal ICA of the third patient re-occluded asymptomatically, as demonstrated by carotid color Doppler ultrasound imaging. Since the overall condition of the patients was good and the risk of treatment was considered to outweigh the potential 
Table I. Baseline characteristics of patients.

\begin{tabular}{|c|c|c|c|}
\hline Variable & SR & no SR & P-value \\
\hline Age (years) & $58.00 \pm 11.1$ & $66.7 \pm 7.38$ & 0.069 \\
\hline $\mathrm{HG}(\mathrm{g} / \mathrm{l})$ & $131.00 \pm 24.56$ & $142.64 \pm 12.76$ & 0.169 \\
\hline $\operatorname{PLT}\left(\times 10^{9} / 1\right)$ & $194.00 \pm 1.73$ & $209.79 \pm 47.70$ & 0.575 \\
\hline Cholesterol (mmol/l) & $3.59 \pm 0.56$ & $4.15 \pm 1.00$ & 0.346 \\
\hline $\mathrm{LDL}(\mathrm{mmol} / \mathrm{l})$ & $1.86 \pm 0.39$ & $2.45 \pm 0.86$ & 0.249 \\
\hline HDL (mmol/l) & $0.84 \pm 0.05$ & $1.09 \pm 0.25$ & 0.100 \\
\hline PT (second) & $11.57 \pm 1.08$ & $11.72 \pm 0.74$ & 0.744 \\
\hline APTT (sec) & $27.13 \pm 6.30$ & $25.94 \pm 4.00$ & 0.638 \\
\hline FIB (g/l) & $3.57 \pm 0.53$ & $3.08 \pm 0.63$ & 0.204 \\
\hline $\mathrm{FG}(\mathrm{mmol} / \mathrm{l})$ & $6.49 \pm 3.68$ & $5.75 \pm 1.70$ & 0.517 \\
\hline Sex (male) & $2(66.7)$ & $31(93.9)$ & 0.236 \\
\hline Ischemic stroke & $2(66.7)$ & $16(48.5)$ & 0.500 \\
\hline Transient ischemic attack & $1(33.3)$ & $17(51.5)$ & 0.500 \\
\hline History of hypertension & $2(66.7)$ & $23(69.7)$ & 0.678 \\
\hline History of diabetes mellitus & $1(33.3)$ & $11(33.3)$ & 0.717 \\
\hline History of coronary heart disease & $1(33.3)$ & $11(33.3)$ & 0.717 \\
\hline Atrial fibrillation & 0 & 0 & \\
\hline Current smoking & $1(33.3)$ & $20(60.6)$ & 0.373 \\
\hline Moderate to heavy drinking & $1(33.3)$ & $16(48.5)$ & 0.543 \\
\hline
\end{tabular}

Values are expressed as the mean \pm standard deviation or $\mathrm{n}(\%)$. SR, patients with late spontaneous recanalization; no SR, patients without late spontaneous recanalization; HG, hemoglobin; PLT, blood platelet; LDL, low-density lipoprotein; HDL, high-density lipoprotein; PT, prothrombin time; APTT, activated partial thromboplastin time; FIB, fibrinogen; FG, fasting glucose.

benefits, no intervention was recommended for these two male patients, and ICA occlusion did not reoccur. None of the three patients with late SR experienced any cerebral vascular event after the confirmation of its occurrence, and all of them had a good overall functional ability. The demographic factors, conventional risk factors of atherosclerosis or cerebral vascular characteristics were not significantly different between patients with and without recanalization. Furthermore, the results of the univariate analyses indicated that none of the above factors were associated with the incidence of late SR of an occluded ICA (Table II). In addition, the occurrence of late SR of the ICA was not associated with the incidence of adverse cardiovascular events $(\mathrm{P}=0.433$, $\mathrm{OR}=2.714$; 95\% CI: 0.223-32.992). Due to the small sample size, statistical comparisons may not be reliable.

\section{Discussion}

In the present prospective cohort study, it was identified that late SR of the ICA occurred in $8.3 \%$ of patients with atherosclerotic ICA occlusion who underwent cerebral vascular stenting for other lesions. Although patients with late SR of the ICA were likely to experience vascular events, these patients generally had a preserved functional ability and a good clinical prognosis. Taken together, the results of the present study suggest that patients with late SR of the ICA appear to have preserved functional ability and tend to have favorable clinical outcomes. However, this sample was too small to identify trends.
The present study has certain strengths compared to various previously published studies on the clinical characteristics of late SR. Patients who underwent percutaneous cerebral stenting of a stenotic cerebral artery other than the occluded ICA were included. Since one or more cerebral arteries with stenosis were accompanied by an occluded ICA, the most likely etiology for ICA occlusion of these included patients was atherosclerosis. A systematic follow-up was performed according to the predefined protocol and information regarding the follow-up was prospectively collected. The results of the present study indicated a relatively higher incidence of late SR when compared with previously reported ones. Previous studies on the clinical characteristics of SR were generally based on case reports and case series with the rate of recanalization ranging from 2 to $68 \%(1-3,6,9,18)$. The incidence of SR of an occluded ICA caused by dissection was higher than that caused by atherosclerotic or embolic events $(6,19)$, and the incidence of early SR was higher than that of late SR $(2,9,10,18)$. Reasons for the potential underestimated incidence of late SR in previous studies may be due to the difficulty of long-term follow-up for cerebral arteries with valid imaging examinations and the different etiologies of cerebral artery occlusion.

The potential mechanisms underlying the pathogenesis of late SR remain largely elusive. It has been suggested that early SR may occur via various mechanisms, including endogenous clot lysis, temporary edema of the arterial wall, intraplaque hemorrhage, regression of endothelial edema, simply missed pseudo-occlusions, spontaneous fibrinolysis or lytic capabilities 
of the endothelium $(1,20)$. In addition, according to previous studies, late SR may be caused by the fibrinolytic system or the presence of vasa vasorum, providing collateral circulation $(11,21)$. Obviously, further studies are required to determine the exact mechanisms. In the present study, based on the string flow after recanalization of the ICA, it was speculated that the SR was caused by the fibrinolytic system or regression of endothelial edema. In addition, due to the lack of a good flow, the ICA lumen was vulnerable to re-occlusion. The third patient of the present study presented with asymptomatic recanalization at 16 months after onset, and presented with an asymptomatic re-occlusion 6 months thereafter. If follow-ups by carotid color Doppler ultrasound imaging had been performed less frequently, the recanalization of the third patient would not have been detected. It may be speculated that more frequent follow-ups allow for the detection of more cases of recanalization.

In the present study, all three patients with late SR had vascular events that did not lead to disability immediately prior to the confirmation of SR. However, none of these patients had cerebral vascular events since the SR of the occluded ICA, and all of them had a relatively preserved functional ability and favorable clinical outcomes. These results are consistent with those of previous studies, which also indicated a favorable clinical prognosis for patients with late SR of cerebral arteries. In a study by Camporese et al (9), SR occurred in $2.3 \%$ of patients with ICA occlusion of embolic origin, who were complaint-free throughout the observation period. Furthermore, a study by Delgado et al (10) included 7 patients with late SR among 182 patients with symptomatic occlusion of the ICA; 6 of these patients were stable since then and 1 died of cancer that was not associated with atherosclerosis. Of note, certain studies have indicated that ICA occlusion is associated with considerable risks: Verlato et al (22) demonstrated that the stroke-associated mortality rate in patients with ICA occlusion was $7.3 \%$, and a study by Inzitari et al (23) indicated that ICA stenosis without recanalization was associated with a risk of ipsilateral stroke of $60-99 \%$, the occurrence rate of which was $9.4 \%$ over 5 years. The two studies revealed worse outcomes for patients with ICA without SR vs. with late SR. The potential reasons for certain differences between the abovementioned studies, including the present one, may be multifactorial. Numerous differences may be involved, including baseline characteristics, the severity of cerebral vascular lesions and potential treatments. These should be clarified in future studies with a large sample size.

The best treatment to achieve recanalization of the ICA remains to be determined. In previous studies, carotid endarterectomy has been frequently performed on patients who presented with SR of the ICA (10-15). However, since the natural course and outcome of SR of an occluded ICA appears to be relatively favorable, medication treatment appears to be adequate. If the stenosis of the recanalized artery is not too long, other therapeutic approaches besides medication treatment may be performed, but the potential risks should be carefully evaluated. In the present study, medication treatment was recommended for all 3 patients with SR, and the clinical outcomes of these patients were optimal.

Since patients with SR of ICA usually have favorable long-term outcomes, it is of significance to identify the potential
Table II. Association between cardiovascular risk factors and late spontaneous recanalization (univariate analysis).

\begin{tabular}{lclc}
\hline Variable & OR & \multicolumn{1}{c}{$95 \%$ CI } & P-value \\
\hline Age & 0.933 & $0.791-1.100$ & 0.407 \\
HG & 1.032 & $0.939-1.134$ & 0.513 \\
PLT & 1.001 & $0.976-1.027$ & 0.930 \\
Cholesterol & 0.634 & $0.150-2.685$ & 0.536 \\
LDL & 0.616 & $0.121-3.125$ & 0.559 \\
HDL & 1.973 & $0.015-263.952$ & 0.786 \\
PT & 0.754 & $0.145-3.902$ & 0.736 \\
APTT & 1.073 & $0.807-1.426$ & 0.629 \\
FIB & 3.062 & $0.525-17.861$ & 0.214 \\
FG & 0.173 & $0.016-1.927$ & 0.154 \\
Sex & 7.750 & $0.474-126.693$ & 0.151 \\
NIHSS & 1.000 & $0.671-1.490$ & 1.000 \\
Ischemic stroke & 0.471 & $0.039-5.708$ & 0.554 \\
History of hypertension & 0.870 & $0.705-10.728$ & 0.913 \\
History of coronary & 1.000 & $0.081-12.270$ & 0.717 \\
heart disease & & & \\
Current smoking & 0.325 & $0.027-3.959$ & 0.378 \\
Moderate to heavy & 0.531 & $0.044-6.444$ & 0.619 \\
drinking & & & \\
\hline
\end{tabular}

OR, odds ratio; CI, confidence interval; HG, hemoglobin; PLT, blood platelets; LDL, low-density lipoprotein; HDL, high-density lipoprotein; PT, prothrombin time; APTT, activated partial thromboplastin time; FIB, fibrinogen; FG, fasting glucose; NIHSS, National Institute of Health Stroke Scale.

Table III. Characteristics of the patients with ICA occlusion and clinical outcomes during follow-up.

\begin{tabular}{lc}
\hline Variable & $\mathrm{n}(\%)$ \\
\hline Side of the occluded ICA (right) & $24(68.6)$ \\
Additional non-ICA stenosis & \\
Vertebral artery & $22(61.1)$ \\
Carotid artery other than ICA & $25(69.4)$ \\
Intracranial artery & $15(41.7)$ \\
Symptomatic occlusion & $22(61.1)$ \\
Vascular events & $16(44.4)$ \\
Recurrent stroke & $9(25.0)$ \\
Cardiovascular events & $7(19.4)$ \\
Death & $2(5.6)$ \\
Spontaneous recanalization & $3(8.3)$ \\
Modified Ranking Scale $\geq 3$ & $5(13.9)$
\end{tabular}

Vascular events in this study include recurrent stroke and cardiovascular events. ICA, internal carotid artery.

predictors for the SR of an occluded ICA. In the present study, a significant association between the clinical characteristics and the occurrence of late SR in these patients was not 
established, which was probably due to the limited number of included patients with SR. Furthermore, Xu et al (18) revealed that atrial fibrillation is associated with a reduced incidence of SR, whereas stage 3 hypertension may promote this. However, the etiology of ICA occlusion was not unified in that study, and the follow-up period was only 3 months. Studies with a large sample size and long follow-up durations are required to confirm the present results.

The present study has certain limitations that should be considered when interpreting the results. First, the number of subjects enrolled was relatively small, and the statistical power was probably insufficient to detect a significant association between clinical characteristics and the incidence of SR. Furthermore, color-coded Doppler ultrasound imaging is not the gold standard for determining the degree of carotid stenosis and occlusion. However, this was used as the primary examination during the follow-up, as it is the most effective non-invasive screening method for the carotid artery.

In conclusion, in line with previous observations, the present study suggested that patients with late SR of the ICA tend to have preserved functional ability and favorable clinical outcomes. Large-scale cohort studies are required to confirm these results and determine the clinical characteristics that contribute to the incidence of late SR of ICA. Retrospective studies or meta-analyses may be suitable further elucidate this.

\section{Acknowledgements}

Not applicable.

\section{Funding}

No funding was received.

\section{Availability of data and materials}

The datasets used and/or analyzed during the current study are available from the corresponding author on reasonable request.

\section{Authors' contributions}

HW performed the registration of hospitalization data for all patients and was a major contributor in writing the manuscript. YL designed the study and performed the registration of follow-up data for all patients. BL and CL clinically evaluated all patients and completed carotid stenting. $\mathrm{HZ}$ and $\mathrm{HW}$ analyzed and explained data on hospitalization and follow-up for all patients. All authors read and approved the final manuscript.

\section{Ethics approval and consent to participate}

The study was approved by the Ethics Committee of the Affiliated Yantai Yuhuangding Hospital of Qingdao University. All patients provided written informed consent to participate in the study.

\section{Patient consent for publication}

Not applicable. 


\section{Competing interests}

The authors declare that they have no competing interests.

\section{References}

1. Meves SH, Muhs A, Federlein J, Buttner T, Przuntek H and Postert T: Recanalization of acute symptomatic occlusions of the internal carotid artery. J Neurol 249: 188-192, 2002.

2. Nguyen-Huynh MN, Lev MH and Rordorf G: Spontaneous recanalization of internal carotid artery occlusion. Stroke 34 1032-1034, 2003.

3. Tuskan-Mohar L, Legac M, Bralic M and Budiselic B: Spontaneous recanalization of internal carotid artery occlusion. Wiener klinische Wochenschrift 122: 633-636, 2010.

4. Zhou Z, Qian D, Liu L, Tang WX, Jia SH and Liu ZJ Morphological change of early spontaneous recanalization following internal carotid artery occlusion due to possible dissection. Chin Med J 129: 2757-2758, 2016.

5. Bogousslavsky J, Despland PA and Regli F: Spontaneous carotid dissection with acute stroke. Arch Neurol 44: 137-140, 1987.

6. Steinke W, Rautenberg W, Schwartz A and Hennerici M: Noninvasive monitoring of internal carotid artery dissection. Stroke 25: 998-1005, 1994.

7. Akiyama Y, Tanaka M, Hayashi J, Fujimoto M, Harada K, Nakahara I and Taniwaki Y: Internal carotid artery obstruction derived from persistent arterial wall dissection associated with old trivial trauma. Neurol Med Chir 46: 395-397, 2006.

8. Sturzenegger M, Mattle HP, Rivoir A and Baumgartner RW: Ultrasound findings in carotid artery dissection: Analysis of 43 patients. Neurology 45: 691-698, 1995.

9. Camporese G, Labropoulos N, Verlato F, Bernardi E, Ragazzi R, Salmistraro G, Kontothanassis D, Andreozzi GM and Carotid Recanalization Investigators Group: Benign outcome of objectively proven spontaneous recanalization of internal carotid artery occlusion. J Vasc Surg 53: 323-329, 2011.

10. Delgado MG, Vega PP, Lahoz CH and Calleja S: Late spontaneous recanalization of symptomatic atheromatous internal carotid artery occlusion. Vascular 23: 211-216, 2015.

11. Buslovich S and Hines GL: Spontaneous recanalization of chronic internal carotid artery occlusions: Report of 3 cases. Vasc Endovascular Surg 45: 93-97, 2011.
12. Klonaris C, Alexandrou A, Katsargyris A, Liasis N and Bastounis E: Late spontaneous recanalization of acute internal carotid artery occlusion. J Vasc Surg 43: 844-847, 2006.

13. Matic P, Ilijevski N, Radak S, Kolar J and Radak D: Recanalization of chronic carotid occlusion: Case report and review of the literature. Vascular 17: 281-283, 2009.

14. Som S and Schanzer B: Spontaneous recanalization of complete internal carotid artery: A clinical reminder. J Surg Tech Case Rep 2: 73-74, 2010.

15. Shah PS, Hingorani A, Ascher E, Shiferson A, Patel N and Gopal K: Spontaneous recanalization of an occluded internal carotid artery. Ann Vasc Surg 24: e1-e4, 2010.

16. National Institute of Neurological D and Stroke rt PASSG: Tissue plasminogen activator for acute ischemic stroke. N Engl J Med 333: 1581-1587, 1995.

17. Quinn TJ, Lees KR, Hardemark HG, Dawson J and Walters MR: Initial experience of a digital training resource for modified Rankin scale assessment in clinical trials. Stroke 38: 2257-2261, 2007.

18. Xu Y, Qian G, Wei L, Qin-Hua W, Bo D, Cheng-Chun L, Zhi-Hong Z, Li-Li Z, Zhi-Qiang X, Hua-Dong, et al: Predictive factors for the spontaneous recanalization of large and middle cerebral arteries after acute occlusion. J Stroke Cerebrovasc Dis 25: 1896-1900, 2016.

19. Guillon B, Levy C and Bousser MG: Internal carotid artery dissection: An update. J Neurol Sci 153: 146-158, 1998.

20. Kniemeyer HW, Voshege M, Soliman A, Abu Al Nasr TM, Beckmann $\mathrm{H}$ and Edelmann M: Late spontaneous recanalization of a symptomatically occluded internal carotid artery two years after extra-intracranial bypass. J Cardiovasc Surg 53: 241-246, 2012.

21. Lammie GA, Sandercock PA and Dennis MS: Recently occluded intracranial and extracranial carotid arteries. Relevance of the unstable atherosclerotic plaque. Stroke 30: 1319-1325, 1999.

22. Verlato F, Camporese G, Bernardi E, Salmistraro G, Rocco S, Mayellaro V, Busonera F and Andreozzi GM: Clinical outcome of patients with internal carotid artery occlusion: A prospective follow-up study. J Vasc Surg 32: 293-298, 2000.

23. Inzitari D, Eliasziw M, Gates P, Sharpe BL, Chan RK, Meldrum HE and Barnett HJ: The causes and risk of stroke in patients with asymptomatic internal-carotid-artery stenosis. North American Symptomatic Carotid Endarterectomy Trial Collaborators. N Engl J Med 342: 1693-1700, 2000. 